\title{
On some kinds of dense orbits in general nonautonomous dynamical systems
}

https://doi.org/10.1515/msds-2020-0116

Received October 25, 2019; accepted October 14, 2020

\begin{abstract}
We study the theory of universality for the nonautonomous dynamical systems from topological point of view related to hypercyclicity. The conditions are provided in a way that Birkhoff transitivity theorem can be extended. In the context of generalized linear nonautonomous systems, we show that either one of the topological transitivity or hypercyclicity give sensitive dependence on initial conditions. Meanwhile, some examples are presented for topological transitivity, hypercyclicity and topological conjugacy.
\end{abstract}

Keywords: Birkhoff transitivity theorem, Hypercyclicity, Topological conjugacy

MSC: 37D45, 37B55, 57R18

\section{Introduction}

Nonautonomous dynamical systems provide a successful framework to describe a large variety of phenomena in biology, informatics, economy, medicine, quantum mechanics and etc $[2,8,9,17]$, in the case that they can not be modelled by autonomous systems. A knowledge of the topological and geometrical structure of nonautonomous discrete system is useful both in developing theory and in applications $[6,10]$. In the last decade chaotic behaviour of nonautonomous discrete systems attracted many researchers $[1,3,14,16,18]$. Chaos theory deals with complex and unpredictable behaviour of phenomena over time. Even in the context of dynamical systems, different definitions of chaos has been posed which some of them are classified by Forti [4]. In most of the definitions, topological transitivity plays a central role. In this context, Birkhoff transitivity theorem establishes a straight relationship between topological transitivity and density of orbits on a separable complete metric space without isolated points [5, 13].

The present paper focuses on the generalization of nonautonomous dynamical systems and challenges some topological and dynamical characteristics like topological transitivity, sensitivity, hypercyclicity and conjugacy. Under suitable assumptions, some new results have been achieved that Birkhoff transitivity theorem is one of them. Suppose that $X$ and $X_{0}$ are two nonempty topological spaces and $f_{n}: X_{0} \rightarrow X, n \in \mathbb{N}$ are maps. The orbit of $x_{0} \in X_{0}$ is defined as $\operatorname{orb}\left(x_{0}\right):=\left\{f_{n}\left(x_{0}\right) \mid n \in \mathbb{N}\right\}$. When $\operatorname{orb}\left(x_{0}\right)$ is a dense subset of $X$, the point $x_{0}$ is called universal [5]. We call the sequence $\left\{f_{n}\right\}_{n=1}^{\infty}$ a generalized nonautonomous discrete system (for short GNDS) on $X_{0}$, that determines an equation in the form

$$
x_{n+1}=f_{n}\left(x_{0}\right) .
$$

The definition of a GNDS on $X_{0}$ is more general than the definition of a NDS introduced in $[7,15]$ which is as follows: assume that $X$ is a metric space and for each $n \in \mathbb{N} \cup\{0\}, X_{n}$ is its subspace. Also, let $g_{n}: X_{n} \rightarrow X_{n+1}$ be an arbitrary map, then they call the family $\left\{g_{n}\right\}_{n=0}^{\infty}$ a NDS. Indeed, such a family generates a NDS via time-dependent difference equations $x_{n+1}=g_{n}\left(x_{n}\right)$. Here, for a given element $x_{0} \in X_{0}$, a sequence $\left\{x_{n}\right\}_{n=0}^{\infty}$ is called the orbit of $x_{0}$ (or an orbit of $\left\{g_{n}\right\}_{n=0}^{\infty}$ in the iterative way) and is defined as

$$
x_{0}, x_{1}=g_{0}\left(x_{0}\right), x_{2}=g_{1}\left(x_{1}\right), \ldots, x_{n+1}=g_{n}\left(x_{n}\right), \ldots
$$

\footnotetext{
`Corresponding Author: Mehdi Pourbarat: Dep. Math., Shahid Beheshti University, Tehran, Iran
} 
Consider $f_{n}:=g_{n-1} \circ g_{n-2} \cdots \circ g_{0}$, we observe that $f_{n}$ is a map from $X_{0}$ to $X$, and therefore our definition is a generalization (see Example 1.(h) and the explanation after that).

Throughout the paper, we always assume that $X_{0}$ is a subspace of $X$. Hence, for given $x \in X_{0}$ one may expect two main types of density by restricting to the subspace $X_{0}$. In the other word, for the underlying GNDS we always deal with the subsets

$$
O_{1}:=\left\{x \in X_{0} \mid \overline{\operatorname{arb}(x) \cap X_{0}}{ }^{X_{0}}=X_{0}\right\}, \quad O_{2}:=\left\{x \in X_{0} \mid \overline{\operatorname{orb}(x)}^{X} \cap X_{0}=X_{0}\right\} .
$$

Investigating topological structure of these subspaces leads us to achieve some results that are organized as follows:

In Section 2, some properties of subspaces $O_{1}$ and $O_{2}$ are discussed, and then we challenge these subspaces with topological transitivity. In fact we prove that if $\overline{O_{1}}=X_{0}$, the system is topologically transitive. Also, under certain conditions for underlying spaces and maps $f_{n}$ (see Theorem 2), the topological transitivity yields $\overline{O_{2}}=X_{0}$.

In Section 3, the concepts of topological $h$-conjugacy and topological $\left\{h_{n}\right\}$-conjugacy are defined for GNDSs on $X_{0}$. We show that these two definitions fit exactly to Definition 3.6 in [16] and Definition 3.1 in [15], respectively. Then considering the hypothesis $h_{n}$ some classical dynamical properties are preserved under two $\left\{h_{n}\right\}$-conjugated equations.

In Section 4, assuming underlying space $X$ as a Frechet space we focus on the generalized linear nonautonomous discrete systems (in short GLNDSs). Indeed we prove that either topological transitivity or hypercyclicity always implies sensitive dependence on initial conditions. Also, in this section two examples are posed, the first system is topologically transitive while $\overline{O_{1}} \neq X_{0}$, and for the second one (that satisfies hypothesis of Theorem 2) $\overline{O_{2}}=X_{0}$ holds while it is not topologically transitive.

In the study of dynamical systems, we may deal with results that less hypothesis for their proofs can be written. That is why we choose the underling system in the general form, and then we provide the conditions for the system in a way that some classical results obtained. As we mentioned in above, these results are related to chaos, topological conjugacy and linear systems. To continue this, it seems that we can find more results around expansiveness, sensitivity and topological entropy. Another extension is to interpret a kind of closeness for two general nonautonomous dynamical systems, and then to achieve the results in some contexts like symbolic dynamic, bifurcation theory and etc.

\section{Hypercyclic Orbits and Topological Transitivity}

We begin the section by posing the definition of a GNDS.

Definition 1. Let $X$ be a topological space and $X_{0}$ be a subspace of $X$. A sequence $\left\{f_{n}\right\}_{n=1}^{\infty}$ of maps $f_{n}: X_{0} \rightarrow X$ is called a GNDS on $X_{0}$ and the orbit of $x_{0} \in X_{0}$ is defined as $\operatorname{orb}\left(x_{0}\right):=\left\{f_{n}\left(x_{0}\right) \mid n \in \mathbb{N}\right\}$.

We write $f_{1, \infty}$ instead of $\left\{f_{n}\right\}_{n=1}^{\infty}$. Obviously, a dynamical system $g: X \rightarrow X$ can be considered as a GNDS on $X_{0}$ by setting $f_{n}:=g^{n}{ }_{\mid X_{0}}$. Many other examples are presented here that may be useful in the study of the theory of GNDSs.

Example 1. (a) Let $Y$ be a topological space and $F: X_{0} \times Y \rightarrow X$ be an arbitrary map. Also, let $\left\{a_{n}\right\}_{n=1}^{\infty}$ be a sequence in $Y$. Then $f_{1, \infty}$ given by $f_{n}(x)=F\left(x, a_{n}\right)$ is a GNDS on $X_{0}$.

(b) Let $M$ be a smooth manifold and $f \in \operatorname{Diff}(M)$. Also, let $p$ be a hyperbolic point and $X_{0}=W_{l o c}^{u}(p)$. Then $f_{1, \infty}$ given by $f_{n}(x)=f^{n}(x)$ is a GNDS on $W_{l o c}^{u}(p)$. The similar situation can be written for $W_{l o c}^{s}(p)$.

(c) Let $g_{1}$ and $g_{2}$ be two discrete dynamical systems on $X$ and $A$ be any subset of natural numbers. Then $f_{1, \infty}$ given by 


$$
f_{n}(x)=\left\{\begin{array}{ll}
g_{1}^{n}(x) & n \in A \\
g_{2}^{n}(x) & n \notin A
\end{array}, \quad x \in X_{0}\right.
$$

is a GNDS on $X_{0}$.

(d) Let $\left\{\alpha_{n}\right\}_{n=1}^{\infty}$ be a sequence of real numbers. Then $f_{1, \infty}$ given by $f_{n}(x)=\alpha_{n} x(1-x)$ is a GNDS on $[0,1]$. We call it Logistic GNDS corresponding to the sequence $\left\{\alpha_{n}\right\}_{n=1}^{\infty}$.

(e) Let $\mathbb{H}(\mathbb{C})$ be a set of all holomorphic functions from $\mathbb{C}$ into itself and $\left\{g_{n}\right\}_{n=0}^{\infty}$ be a sequence in $\mathbb{H}(\mathbb{C})$. Also, let $D$ be the differentiable operator and maps $f_{n}$ defined by

$$
f_{n}(g)=\sum_{k=0}^{n} \frac{D^{k} g(0)}{k !} g_{k}
$$

Then $f_{1, \infty}$ is a GLNDS on any given subspace $X_{0}$ of $\mathbb{H}(\mathbb{C})$. Notice that in the case $g_{n}(x):=x^{n}, f_{n}(g)$ is the map that associates with $g$ its Taylor polynomial of degree $n$ at 0 .

(f) Let $\left\{\alpha_{n}\right\}_{n=1}^{\infty}$ be a sequence of real numbers and $X$ be the space of all continuous functions on $\mathbb{R}$ endowed with the sup norm. Also, let $f_{n}(g)=\tilde{g}$ where $\tilde{g}$ is Birkhoff's translation $\tilde{g}(x):=g\left(x+\alpha_{n}\right)$. Then $f_{1, \infty}$ is a GLNDS on the subspace $X_{0}$.

(g) Let $\left\{\alpha_{n}\right\}_{n=1}^{\infty}$ be a sequence of real numbers. For each $1 \leq p<\infty$, let $X:=l^{p}$ be the space of all $\mathrm{p}$ summable sequences. Then $f_{1, \infty}$ given by

$$
f_{n}\left(\left(x_{1}, x_{2}, \cdots, x_{n}, \cdots\right)\right)=\alpha_{n}\left(x_{n}, x_{n+1}, \cdots\right)
$$

is a linear GNDS on any subspace $X_{0}$ of $l^{p}$.

(h) Let $f_{n}:[0,1) \rightarrow[0,1)$ be given by $f_{n}(x)=n x \bmod 1$. Then $f_{1, \infty}$ is a GNDS on $[0,1)$.

In general a GNDS can not be stated in the iterative way, but if all maps $f_{n}$ are bijective, by getting $g_{0}:=f_{1}$ and $g_{n}:=f_{n+1} \circ f_{n}^{-1}$ as the maps from $X_{n}:=f_{n}\left(X_{0}\right)$ to $X_{n+1}:=f_{n+1}\left(X_{0}\right)$ then this GNDS is in the iterative way.

In the case that the maps $f_{n}$ (even one of them) are not injective, maybe GNDS can not be stated in the iterative way. For instance, in Example 1.(h) there is no function $g_{n}: X_{n} \rightarrow X_{n+1}$ with $X_{n}=X_{n+1}=[0,1)$ such that $g_{n} \circ f_{n}=f_{n+1}$.

To achieve this, let $A_{n}:=\left\{a \mid f_{n}(a)=\frac{1}{2}\right\}$. Thus, $A_{n}=\left\{\frac{2 k+1}{2 n} \mid 0 \leq k<n, k \in \mathbb{N} \cup\{0\}\right\}$. Hence, for every $0 \leq k<n$ one follows:

$$
g_{n} \circ f_{n}\left(\frac{2 k+1}{2 n}\right)=g_{n}\left(\frac{1}{2}\right), \quad f_{n+1}\left(\frac{2 k+1}{2 n}\right)=\frac{n+1}{2 n}(2 k+1) \bmod 1 .
$$

Consequently, $g_{n}\left(\frac{1}{2}\right)=\frac{n+1}{2 n}(2 k+1) \bmod 1$, which is in contradiction with the well-definitive of the function $g_{n}$.

The subset $A \subset X_{0}$ is said to be invariant if $f_{n}(A) \subset A$ for every $n \in \mathbb{N}$. The point $x_{0}$ is a fixed point if $\left\{x_{0}\right\}$ be invariant. It is called weakly $k$-periodic if $f_{m k}\left(x_{0}\right)=x_{0}$, for every $m \in \mathbb{N}$. The smallest such $k$ is called the period of $x_{0}$ and the set of weakly periodic points is denoted by $W P\left(f_{n}\right)$. Also, weakly $k$-periodic point $x_{0}$ is called $k$-periodic if $f_{m k+n}\left(x_{0}\right)=f_{n}\left(x_{0}\right)$, for every $m, n \in \mathbb{N}$. The set of periodic points is denoted by $P\left(f_{n}\right)$, and so $P\left(f_{n}\right) \subset W P\left(f_{n}\right)$. The point $x_{0}$ is called a finite orbit point if the set $\operatorname{orb}\left(x_{0}\right)$ is finite. The set of finite orbit points is denoted by $\operatorname{Fin}\left(f_{n}\right)$. Notice that, $P\left(f_{n}\right) \subset \operatorname{Fin}\left(f_{n}\right)$ while the following example demonstrates that in general the relation $W P\left(f_{n}\right) \subset \operatorname{Fin}\left(f_{n}\right)$ does not hold.

Example 2. Consider $f_{n}(x)=\left\{\begin{array}{l}\frac{x}{2}\left(n+1+(-1)^{n}(1-n)\right) \quad x>0 \\ -x \quad x \leqslant 0\end{array}\right.$. The point 0 is the only fixed point for GNDS $f_{1, \infty}$. It is easy to verify that $P\left(f_{n}\right)=\{0\}, W P\left(f_{n}\right)=\mathbb{R}^{+} \cup\{0\}$ and $\operatorname{Fin}\left(f_{n}\right)=\mathbb{R}^{-} \cup\{0\}$.

Now we are going to generalize some topological concepts and results that is stated in [11], for GNDSs on $X_{0}$ and discuss the complicated behaviour that appears in the universality process. There are two kinds of densities of orbits for GNDSs on $X_{0}$ as follows: 
- GNDS $f_{1, \infty}$ is called hypercyclic or universal on $X_{0}$, if there is $x_{0} \in X_{0}$ such that $\operatorname{orb}\left(x_{0}\right) \cap X_{0}$ is dense in $X_{0}$. In such a case, $x_{0}$ is called a hypercyclic point and the set of hypercyclic points is denoted by

$$
O_{1}:=\left\{x \in X_{0} \mid \overline{\operatorname{orb}(x) \cap X_{0}}{ }^{X_{0}}=X_{0}\right\} .
$$

- GNDS $f_{1, \infty}$ is called weakly hypercyclic or weakly universal on $X_{0}$, if there exists a point whose orbit's closure contains $X_{0}$. Such a point is called a weakly hypercyclic point and the set of weakly hypercyclic points is denoted by

$$
O_{2}:=\left\{x \in X_{0} \mid \overline{\operatorname{orb}(x)}^{X} \cap X_{0}=X_{0}\right\}
$$

Thus, in dealing with the terminology "density of an orbit" we must specify which one of the above subspaces is intended. When these sets are large subspaces from topological point of view, we will expect a rich dynamic around $X_{0}$. Before going any further, it is important to know the relationship between $O_{1}$ and $O_{2}$.

Proposition 1. With notations as above, we have:

i) $O_{1}=\left\{x \in X_{0} \mid \quad{\overline{\operatorname{orb}(x) \cap X_{0}}}^{X} \supseteq X_{0}\right\}$,

ii) $\mathrm{O}_{1} \subseteq \mathrm{O}_{2}$,

iii) if $X_{0}$ is an open set in $X$, then $O_{1}=O_{2}$.

Proof. The same proof of Theorem 1 in [11].

Thus, every hypercyclic point is a weak hypercyclic point since $O_{1} \subset O_{2}$. The sets $O_{1}$ and $O_{2}$ emerged in the following example are non trivial subsets of $X_{0}$ and $O_{1} \neq O_{2}$.

Example 3. Let $X=\mathbb{R}^{4}$ and $X_{0}=\mathbb{R}^{3} \times\{0\}$. Let $\left\{\left(r_{n}, s_{n}, t_{n}\right)\right\}_{n=1}^{\infty}$ be a dense subsequence in $\mathbb{R}^{3}$ and $a_{1}, a_{2}, \ldots, a_{10}$ ten arbitrary constants. Consider the GLNDS $T_{1, \infty}$ given by $T_{n}(x)=A_{n} x$, where

$$
A_{n}=\left[\begin{array}{cccc}
r_{n} & a_{1} & a_{2} & a_{3} \\
s_{n} & a_{4} & a_{5} & a_{6} \\
t_{n} & a_{7} & a_{8} & a_{9} \\
0 & \frac{1}{n} & n & a_{10}
\end{array}\right]
$$

Then $O_{1}=\mathbb{R}^{\star} \times\{(0,0,0)\}$ and $O_{2}=\mathbb{R}^{\star} \times \mathbb{R} \times\{(0,0)\}$. Indeed, the assertions follow immediately from equalities

$$
A_{n}\left[\begin{array}{l}
x \\
0 \\
0 \\
0
\end{array}\right]=\left[\begin{array}{c}
r_{n} x \\
s_{n} x \\
t_{n} x \\
0
\end{array}\right], \quad A_{n}\left[\begin{array}{l}
x \\
y \\
0 \\
0
\end{array}\right]=\left[\begin{array}{c}
r_{n} x+a_{1} y \\
s_{n} x+a_{4} y \\
t_{n} x+a_{7} y \\
\frac{y}{n}
\end{array}\right], \quad A_{n}\left[\begin{array}{c}
x \\
y \\
z \\
0
\end{array}\right]=\left[\begin{array}{c}
r_{n} x+a_{1} y+a_{2} z \\
s_{n} x+a_{4} y+a_{5} z \\
t_{n} x+a_{7} y+a_{8} z \\
\frac{y}{n}+n z
\end{array}\right],
$$

where $x, y, z \in \mathbb{R}$. Notice that, for all the points $(x, y, z, 0) \in X_{0}$ with $z \in \mathbb{R}^{\star}$, we always have $\lim _{n \rightarrow \infty}\left|T_{n}(x, y, z, 0)\right|=\infty$.

In the above example, if all the matrixs $A_{n}$ are invertible, then by taking $g_{n}:=A_{n+1} A_{n}^{-1}$ as the functions from $A_{n} X_{0}$ to $A_{n+1} X_{0}$, this GNDS is stated in the iterative way (see the explanation after Example 1).

Topological transitivity can be extended as below (see Definition 2.3 in [16]).

Definition 2. GNDS $f_{1, \infty}$ is called topologically transitive on $X_{0}$ if for any two nonempty open sets $U_{0}$ and $V_{0}$ of $X_{0}$, there exists some $n \in \mathbb{N}$ such that $f_{n}\left(U_{0}\right) \cap V_{0} \neq \phi$.

Now we are going to state the relation between density and topological transitivity.

Theorem 1. If $\overline{O_{1}}=X_{0}$, then GNDS $f_{1, \infty}$ is topologically transitive on $X_{0}$. 
Proof. Let $U_{0}$ and $V_{0}$ be non-empty open subsets of $X_{0}$. Since $O_{1}$ is dense in $X_{0}$, there exists $x \in U_{0} \cap O_{1}$ such that $\operatorname{orb}(x) \cap X_{0}$ is dense in $X_{0}$. This implies the existence of $n \in \mathbb{N}$ such that $f_{n}(x) \in V_{0}$. Hence, $f_{n}\left(U_{0}\right) \cap V_{0} \neq \phi$ which completes the proof.

The GLNDS appeared in Example 3 is hypercyclic but not topologically transitive. Indeed, Theorem 1 expresses that to have topological transitivity property, we need all hypercyclic points to be dense in $X_{0}$. Unfortunately, topological transitivity does not imply even density of $\mathrm{O}_{2}$ in general. The next theorem expresses that under suitable assumptions, if a GNDS is topologically transitive, then $\mathrm{O}_{2}$ forms a large subspace from topological point of view.

Theorem 2. Let $X_{0}$ be second countable and Baire subspace of $X$, and $X$ be first countable at any point of $X_{0}$. Also, suppose that all $f_{n}$ s are continuous. If GNDS $f_{1, \infty}$ is topologically transitive on $X_{0}$, then $\overline{O_{2}}=X_{0}$ and further that $\mathrm{O}_{2}$ is a $G_{\delta}$-set.

Proof. Since $X_{0}$ is a second countable subspace and $X$ is the first countable at any point of $X_{0}$, it is easy to show the existence of a collection $\left\{U_{m}\right\}_{m \in N}$ of open sets in $X$ such that

i) $U_{m} \cap X_{0} \neq \phi$,

ii) the family $\left\{U_{m} \cap X_{0}\right\}_{m \in \mathbb{N}}$ is a basis for $X_{0}$,

iii) for each $x_{0} \in X_{0}$, the family $\left\{U_{m}\right\}_{m \in \mathbb{N}}$ is a local basis for $x_{0}$ in $X$.

We claim that

$$
O_{2}=\bigcap_{m=1}^{\infty} \bigcup_{n=1}^{\infty} f_{n}^{-1}\left(U_{m}\right)
$$

To prove the claim, let $O^{\star}:=\bigcap_{m=1}^{\infty} \bigcup_{n=1}^{\infty} f_{n}^{-1}\left(U_{m}\right)$. First, we show that $O_{2} \subseteq O^{\star}$. Suppose otherwise, there is $x \in O_{2}$ such that $x \notin O^{\star}$. So if $x \notin O^{\star}$, there exists $m \in \mathbb{N}$ such that for each $n \in \mathbb{N}$ we have $f_{n}(x) \notin U_{m}$. Hence, $\operatorname{orb}(x) \cap U_{m}=\phi$. Since $U_{m} \cap X_{0} \neq \phi$, there exists an element $z \in U_{m} \cap X_{0}$, such that $z \notin \overline{\operatorname{orb}(x)}{ }^{X}$. But $z \in X_{0}$ and so $\overline{\operatorname{orb}(x)}^{X} \cap X_{0} \neq X_{0}$. Therefore $x \notin O_{2}$ which contradicts the choice of $x$. Now, we show $O^{\star} \subseteq O_{2}$. Let $x \in O^{\star}$ but $x \notin O_{2}$. So as $x \in O^{\star}$, for each $m \in \mathbb{N}$, there exists $n \in \mathbb{N}$ such that $f_{n}(x) \in U_{m}$. Thus, $\operatorname{orb}(x) \cap U_{m} \neq \phi$. Moreover, the relation $x \notin O_{2}$ indicates that there exists $z \in X_{0}$ such that $z \notin \overline{\operatorname{orb}(x)}^{X}$. Consequently, there exists $U_{k}$ containing $z$, such that $U_{k} \cap \operatorname{orb}(x)=\phi$. This contradicts the fact that $\operatorname{orb}(x) \cap U_{m} \neq \phi$, for each $m \in \mathbb{N}$, that proves our claim. By continuity of $f_{n}$, each set $\bigcup_{n=1}^{\infty} f_{n}{ }^{-1}\left(U_{m}\right)$ is open and because of transitivity, these open sets are dense in $X_{0}$. Thus, $O_{2}=\bigcap_{m=1}^{\infty} \bigcup_{n=1}^{\infty} f_{n}^{-1}\left(U_{m}\right)$ is a dense $G_{\delta}$-set in $X_{0}$, since $X_{0}$ is a Baire space. This also tells us that $\overline{O_{2}}=X_{0}$ and the proof of Theorem is complete.

Theorems 1 and 2 enable us to state a generalization of Birkhoff transitivity theorem.

Theorem 3. Suppose that $X_{0}$ is a second countable and Baire subspace of topological space $X$. Also, suppose that $X$ is first countable at any point of $X_{0}$ and all the maps $f_{n}$ are continuous. Then,

- density of hypercyclic points implies topological transitivity of GNDS $f_{1, \infty}$ on $X_{0}$,

- topological transitivity of GNDS $f_{1, \infty}$ on $X_{0}$ implies density of a $G_{\delta}$-set of all weakly hypercyclic points.

In particular, when $X_{0}=X$ we can drop "weakly" from the statement of theorem.

\section{Topological Conjugacy}

The conjugacy is an important feature that worths to mention in the study of GNDSs. We begin by an extension of Definition 3.6 in [16] in the usual way. 
Definition 3. Let $X$ and $Y$ be two topological spaces and $X_{0} \subset X, Y_{0} \subset Y$. Let $f_{1, \infty}$ and $g_{1, \infty}$ be two GNDSs on $X_{0}$ and $Y_{0}$, respectively. If there exists a homeomorphism $h: X \rightarrow Y$ such that $h \circ f_{n}=g_{n} \circ h$ on $X_{0}$, for each $n \in \mathbb{N}$, then $f_{1, \infty}$ is said to be topologically $h$-conjugate or simply conjugate to $g_{1, \infty}$.

The conjugacy establishes an equivalence relation on GNDSs. It is easy to show that orbits, invariant sets, weakly $k$-periodic points, $k$-periodic points and finite orbit points are preserved by the conjugated GNDSs. Next theorem asserts that hypercyclic points, weakly hypercyclic points and transitivity are preserved under conjugacy too.

Theorem 4. Let $h: X \rightarrow Y$ be a homeomorphism and GNDS $g_{1, \infty}$ be topologically $h$-conjugate to GNDS $f_{1, \infty}$. Then,

i) if $x_{0}$ is a hypercyclic point (resp. weakly hypercyclic point) for $f_{1, \infty}$, then $h\left(x_{0}\right)$ is a hypercyclic point (resp. weakly hypercyclic point) for $g_{1, \infty}$,

ii) if $f_{1, \infty}$ is topologically transitive on $X_{0}$, then $g_{1, \infty}$ is topologically transitive on $Y_{0}$.

Proof. (i) As $x_{0} \in O_{1}$ (resp. $x_{0} \in O_{2}$ ) in $f_{1, \infty}$, thus $\overline{\operatorname{orb}(x) \cap X_{0}}{ }^{X_{0}}=X_{0}$ (resp. $\overline{\text { orb }\left(x_{0}\right)}{ }^{X} \cap X_{0}=X_{0}$ ). Since $h$ is a homeomorphism, we have $\overline{h(\operatorname{orb}(x)) \cap Y_{0}} Y_{0}=Y_{0}$ (resp. $\overline{h\left(\operatorname{orb}\left(x_{0}\right)\right)} Y Y_{0}=Y_{0}$ ). Thus, $h\left(x_{0}\right) \in O_{1}$ (resp. $\left.h\left(x_{0}\right) \in O_{2}\right)$ in $g_{1, \infty}$, since $h\left(\operatorname{orb}\left(x_{0}\right)\right)=\operatorname{orb}\left(h\left(x_{0}\right)\right)$. This completes assertion (i).

(ii) Suppose that $U_{0}$ and $V_{0}$ are two nonempty open subsets of $Y_{0}$. Since, GNDS $f_{1, \infty}$ is topologically transitive on $X_{0}$, there exists a number $n \in \mathbb{N}$ such that $f_{n}\left(h^{-1}\left(U_{0}\right)\right) \cap h^{-1}\left(V_{0}\right) \neq \phi$. Since $h$ is a homeomorphism and GNDS $g_{1, \infty}$ is $h$-conjugate to $f_{1, \infty}$, then

$$
g_{n}\left(U_{0}\right) \cap V_{0}=h \circ f_{n} \circ h^{-1}\left(U_{0}\right) \cap V_{0} \neq \phi .
$$

Thus, GNDS $g_{1, \infty}$ is topologically transitive on $Y_{0}$ that implies the proof for (ii).

Consequently, if GNDS $g_{1, \infty}$ is conjugate to GNDS $f_{1, \infty}$, then for $i=1,2,{\overline{O_{i}}}^{X_{0}}=X_{0}$, if and only if ${\overline{O_{i}}}^{Y_{0}}=Y_{0}$.

In the context of metric spaces, Tian and Chen proved that two uniformly topologically conjugate NDSs share the sensitive dependence on initial conditions (see Definition 5) too. But similar conclusion does not necessarily hold for two conjugate NDSs [16]. As we observed, homeomorphism $h$ plays a key role in transferring the classical dynamical properties of GNDS $f_{1, \infty}$ to GNDS $\left\{g_{n}\right\}$. Since the maps emerged in GNDSs may be selected randomly, one do not expect such homeomorphism always exists, see Example 4. For the moment, let $X^{\star}:=\bigcap_{n=0}^{\infty} X_{n}$ and $Y^{\star}:=\bigcap_{n=0}^{\infty} Y_{n}$. In the lack of a conjugacy between GNDS $\left\{f_{n}\right\}$ and GNDS $\left\{g_{n}\right\}$, the existence of a conjugacy between GNDS $\left\{F_{n}\right\}$ and GNDS $\left\{G_{n}\right\}$ is possible, where $F_{n}:=f_{n \mid X^{*}}$ and $G_{n}:=g_{n \mid Y^{*}}$. In such cases, most of the dynamical properties are permanent for GNDSs $\left\{f_{n}\right\}$ and $\left\{g_{n}\right\}$, see Theorem 5 . This leads us to another type of conjugacy as below, which completely fits into other Definitions. Two conjugated GNDSs in the sense of Definition 4 may share a given dynamical property under special additional assumptions. For the sake of simplicity, let $X_{n}:=f_{n}\left(X_{0}\right)$ and $Y_{n}:=g_{n}\left(Y_{0}\right)$.

Definition 4. Let $X$ and $Y$ be two topological spaces and $X_{0} \subset X, Y_{0} \subset Y$. Let $f_{1, \infty}$ and $g_{1, \infty}$ be two GNDSs on $X_{0}$ and $Y_{0}$, respectively. If there exist homeomorphisms $h_{0}: X_{0} \rightarrow Y_{0}$ and $h_{n}: X_{n} \rightarrow Y_{n}$ such that $h_{n} \circ f_{n}=$ $g_{n} \circ h_{0}$, for each $n \in \mathbb{N}$. Then GNDS $f_{1, \infty}$ is said to be topologically $\left\{h_{n}\right\}$-conjugate or simply $\left\{h_{n}\right\}$-conjugate to GNDS $g_{1, \infty}$.

Obviously, the $\left\{h_{n}\right\}$-conjugacy establishes an equivalent relation on GNDSs. Also, if GNDS $\left\{f_{n}\right\}$ is topologically $h$-conjugate to GNDS $\left\{g_{n}\right\}$, then by taking $h_{0}:=h_{\mid X_{0}}$ and $h_{n}:=h_{\left.\right|_{X_{n}}}$, they are $\left\{h_{n}\right\}$-conjugate. The condition $h_{n} \circ f_{n}=g_{n} \circ h_{0}$ indicates that if $\left\{x_{n}\right\}_{n=0}^{\infty}$ is an orbit of the $\left\{f_{n}\right\}$, then $\left\{h_{n}\left(x_{n}\right)\right\}_{n=0}^{\infty}$ is an orbit of GNDS $\left\{g_{n}\right\}$ that starts from $h_{0}\left(x_{0}\right)$, since

$$
h_{n}\left(x_{n}\right)=h_{n}\left(f_{n}\left(x_{0}\right)\right)=g_{n}\left(h_{0}\left(x_{0}\right)\right) .
$$

Indeed, in the case that all maps $f_{n}$ and $g_{n}$ are bijective, $\left\{h_{n}\right\}$-conjugacy is equivalent to $\left\{x_{n}\right\}_{n=0}^{\infty}$ is an orbit of GNDS $\left\{f_{n}\right\}$ if and only if, $\left\{h_{n}\left(x_{n}\right)\right\}_{n=0}^{\infty}$ is an orbit of GNDS $\left\{g_{n}\right\}$ that starts from $h_{0}\left(x_{0}\right)$. This fact, for instance, 
says that Definition 4 fits into the situation that any two GNDSs are conjugated by the conjugacy notion in [10]. Example 5.1.1 in the book expresses that every difference equation generating a 2-parameter group is conjugated to the trivial identical equation. Precisely, if $\left(\hat{\varphi}_{k}\right)$ and $\left(\hat{\psi}_{k}\right)$ are generators related with difference equation and identical equation, respectively, then they are conjugated via the homeomorphism $T_{k}$ in the sense of Definition 4. For GNDSs stated in the iterative way, $\left\{h_{n}\right\}$-conjugacy is defined in the following.

Assume that $X$ and $Y$ are two metric spaces and for each $n \in \mathbb{N} \cup\{0\}, X_{n}$ and $Y_{n}$ are subspaces of $X$ and $Y$, respectively. Assume that $g_{n}: X_{n} \rightarrow X_{n+1}$ and $\tilde{g}_{n}: Y_{n} \rightarrow Y_{n+1}$ are arbitrary maps. Also, assume that homeomorphisms $h_{n}: X_{n} \rightarrow Y_{n}$ satisfying $h_{n+1} \circ g_{n}=\tilde{g}_{n} \circ h_{n}$, for each $n$. Then NDS $\left\{g_{n}\right\}_{n=0}^{\infty}$ is said to be topologically $\left\{h_{n}\right\}$-conjugate to NDS $\left\{\tilde{g}_{n}\right\}_{n=0}^{\infty}$, see Definition 3.1 in [15]. As we explained in Section 1, by taking $f_{n}:=g_{n-1} \circ g_{n-2} \cdots \circ g_{0}$ and $\tilde{f}_{n}:=\tilde{g}_{n-1} \circ \tilde{g}_{n-2} \cdots \circ \tilde{g}_{0}, f_{1, \infty}$ and $\tilde{f}_{1, \infty}$ are two GNDSs on $X_{0}$ and $Y_{0}$, respectively that are stated in the successive way.

Proposition 2. With above notation, $h_{n+1} \circ g_{n}=\tilde{g}_{n} \circ h_{n}$ for each $n \in \mathbb{N} \cup\{0\}$, if and only if $h_{n} \circ f_{n}=\tilde{f}_{n} \circ h_{0}$ for each $n \in \mathbb{N}$.

Proof. Firstly, assume that $h_{n+1} \circ g_{n}=\tilde{g}_{n} \circ h_{n}$ for each $n \in \mathbb{N} \cup\{0\}$. Thus,

$$
\begin{aligned}
h_{n} \circ f_{n} & =h_{n} \circ g_{n-1} \circ g_{n-2} \circ \cdots \circ g_{0} \\
& =\tilde{g}_{n-1} \circ h_{n-1} \circ g_{n-2} \circ \cdots \circ g_{0}=\cdots \\
& =\tilde{g}_{n-1} \circ \tilde{g}_{n-2} \cdots \circ \tilde{g}_{0} \circ h_{0}=\tilde{f}_{n} \circ h_{0} .
\end{aligned}
$$

To prove the inverse, let $x \in X_{n}$. Then there is $x_{0} \in X_{0}$ such that $f_{n}\left(x_{0}\right)=x$. Thus,

$$
\begin{aligned}
h_{n+1} \circ g_{n}(x) & =h_{n+1} \circ f_{n+1}\left(x_{0}\right) \\
& =\tilde{f}_{n+1} \circ h_{0}\left(x_{0}\right) \\
& =\tilde{g}_{n} \circ \tilde{f}_{n} \circ h_{0}\left(x_{0}\right) \\
& =\tilde{g}_{n} \circ h_{n} \circ f_{n}\left(x_{0}\right)=\tilde{g}_{n} \circ h_{n}(x) .
\end{aligned}
$$

This completes the proof of the proposition.

Thus, Definition 4 is an extension of Definition 3.1 in [15] (as mentioned above). Roughly speaking, if $\left\{g_{n}\right\}_{n=0}^{\infty}$ and $\left\{\tilde{g}_{n}\right\}_{n=0}^{\infty}$ are two NDSs on $X_{0}$ and $Y_{0}$ corresponding to $f_{1, \infty}$ and $\tilde{f}_{1, \infty}$, respectively, then by proposition 2, $\left\{g_{n}\right\}_{n=0}^{\infty}$ is topologically $\left\{h_{n}\right\}$-conjugate to $\left\{\tilde{g}_{n}\right\}_{n=0}^{\infty}$ if and only if, $f_{1, \infty}$ is topologically $\left\{h_{n}\right\}$-conjugate to $\tilde{f}_{1, \infty}$. The following theorem expresses that if homeomorphisms $h_{0}$ and $h_{n}$ agree on the intersection of their domains, then $h_{0}$ transfers elementary properties such as invariant sets, weakly $k$-periodic points, the set $O_{1}$ and transitivity.

Theorem 5. Let GNDS $f_{1, \infty}$ be $\left\{h_{n}\right\}$-conjugate to GNDSs $g_{1, \infty}$ and $h_{n}=h_{0}$ on $X_{0} \cap X_{n}$. Then,

i) if $A \subset X_{0}$ be an invariant subset for $\left\{f_{n}\right\}$, then $h_{0}(A) \subset Y_{0}$ is an invariant subset for $\left\{g_{n}\right\}$,

ii) if $x_{0}$ is a weakly k-periodic point for $\left\{f_{n}\right\}$, then $h\left(x_{0}\right)$ is a weakly k-periodic point for $\left\{g_{n}\right\}$,

iii) if $x_{0}$ is a hypercyclic point for $\left\{f_{n}\right\}$, then $h\left(x_{0}\right)$ is a hypercyclic point for $\left\{g_{n}\right\}$,

iv) if $f_{1, \infty}$ is topologically transitive on $X_{0}$, then $g_{1, \infty}$ is topologically transitive on $Y_{0}$.

Proof. (i) Because, $A \subset X_{0}$ is an invariant subset for $\left\{f_{n}\right\}$. Thus,

$$
g_{n}\left(h_{0}(A)\right)=h_{n}\left(f_{n}(A)\right) \subset h_{n}(A)=h_{0}(A),
$$

for any $n \in \mathbb{N}$. This means that $h_{0}(A) \subset Y_{0}$ is an invariant subset for $\left\{g_{n}\right\}$.

(ii) Because, $x_{0}$ is a weakly $k$-periodic point for $\left\{f_{n}\right\}$, then $f_{m k}\left(x_{0}\right)=x_{0}$, for every $m \in \mathbb{N}$. Thus,

$$
g_{m k}\left(h_{0}\left(x_{0}\right)\right)=h_{m k}\left(f_{m k}\left(x_{0}\right)\right)=h_{m k}\left(x_{0}\right)=h_{0}\left(x_{0}\right),
$$

for every $m \in \mathbb{N}$. This means $h_{0}\left(x_{0}\right)$ is a weakly $k$-periodic point for $\left\{g_{n}\right\}$. 
(iii) Let $y_{0}:=h_{0}\left(x_{0}\right)$ and $U \in \tau_{Y_{0}}$. Then $h_{0}^{-1}(U) \in \tau_{X_{0}}$ and so

$$
\operatorname{orb}\left(x_{0}\right) \cap X_{0} \cap h_{0}^{-1}(U) \neq \phi,
$$

since $x_{0} \in O_{1}$ in $\left\{f_{n}\right\}$. This guarantees the existence of $x_{n} \in \operatorname{orb}\left(x_{0}\right)$ such that $x_{n} \in X_{0} \cap h_{0}^{-1}(U)$. Due to the fact $x_{n} \in X_{0}$ and by the assumption of the theorem, we obtain $h_{n}\left(x_{n}\right)=h_{0}\left(x_{n}\right) \in Y_{0} \cap U$. On the other hand, the sequence $\left\{y_{n}\right\}_{n=0}^{\infty}$ with $y_{n}=h_{n}\left(x_{n}\right)$ forms the same orbit of $g_{1, \infty}$ which starts from $y_{0}=h_{0}\left(x_{0}\right)$. Hence, orb $\left(y_{0}\right) \cap Y_{0} \cap U \neq \phi$ since $y_{n} \in Y_{0} \cap U$. This implies that $y_{0} \in O_{1}$ in $g_{1, \infty}$ and completes the assertion.

(iv) Suppose that $U_{0}$ and $V_{0}$ are two nonempty open subsets of $Y_{0}$. Since, $f_{1, \infty}$ is topologically transitive on $X_{0}$, there exists some $n \in \mathbb{N}$ such that $\phi \neq f_{n}\left(h_{0}^{-1}\left(U_{0}\right)\right) \cap h_{0}^{-1}\left(V_{0}\right) \in X_{n} \cap X_{0}$. Since, $h_{0}$ is a homeomorphism, by the assumption of the theorem, we have

$$
g_{n}\left(U_{0}\right) \cap V_{0}=h_{n} \circ f_{n} \circ h_{0}^{-1}\left(U_{0}\right) \cap V_{0} \neq \phi .
$$

Thus, $g_{1, \infty}$ is topologically transitive on $Y_{0}$ that implies the proof for (ii).

By the hypothesis of Theorem 5, if invariant subset $A$ is dense in $X_{0}, h_{0}(A)$ is an invariant subset and dense in $Y_{0}$. If $W P\left(f_{n}\right)$ (resp. $O_{1}$ in GNDS $f_{1, \infty}$ ) is dense in $X_{0}$, then $W P\left(g_{n}\right)$ (resp. $O_{1}$ in GNDS $g_{1, \infty}$ ) is dense in $Y_{0}$. The following example says that periodic points, finite orbit points and $\mathrm{O}_{2}$ may not remain preserved under a $\left\{h_{n}\right\}$-conjugacy, even for the linear GNDSs stated in the iterative way with the assumption that $h_{n}=h_{0}$ on $X_{0} \cap X_{n}$ and $h_{n}^{-1}=h_{0}^{-1}$ on $Y_{0} \cap Y_{n}$.

Example 4. Let $\left\{\alpha_{n}\right\}_{n=0}^{\infty}$ be a dense sequences of non zero real numbers. Let $X=Y=\mathbb{R}^{2}$ and $X_{0}=Y_{0}=\mathbb{R} \times\{0\}$. For each $n \in \mathbb{N}$, consider the linear maps

$$
f_{n}(x, 0)=\left(x, \frac{1-(-1)^{n}}{2} x\right), \quad g_{n}(x, 0)=\left\{\begin{array}{cc}
\left(\alpha_{\frac{n-1}{2}} x, \frac{x}{n}\right) & n \text { is odd } \\
(x, 0) & n \text { is even }
\end{array} .\right.
$$

There is no homeomorphism $h$ such that the linear GNDS $\left\{f_{n}\right\}$ is h-conjugate to the linear GNDS $\left\{g_{n}\right\}$. Likewise, $h \circ f_{n}(x, 0)=g_{n} \circ h(x, 0)$, for all $x \in \mathbb{R}$, so sending $n$ to $\infty$ makes a contradiction. While GNDS $\{f S n\}$ is $\left\{h_{n}\right\}$ conjugate to GNDS $\left\{g_{n}\right\}$ with $h_{0}=I D$ and $h_{n}(x, y)=g_{n}(x, 0)$. Note that all maps are one to one and this states that one can write the underlying GNDSs in the iterative way. A simple check shows $P\left(f_{n}\right)=\operatorname{Fin}\left(f_{n}\right)=\mathbb{R} \times\{0\}$ and $\mathrm{O}_{2}=\emptyset$ for GNDS $\left\{f_{n}\right\}$.

Also, for GNDS $\left\{g_{n}\right\}, P\left(g_{n}\right)=F i n\left(g_{n}\right)=\{(0,0)\}$ and $O_{2}=\mathbb{R} \times\{0\}-\{(0,0)\}$. These facts say that periodic points, finite orbit points and the set $\mathrm{O}_{2}$ do not remain preserved under given $\left\{h_{n}\right\}$-conjugacy.

Nonetheless, under additional condition on $h_{n}$ s we get below result.

Theorem 6. Let GNDS $f_{1, \infty}$ be $\left\{h_{n}\right\}$-conjugate to GNDS $g_{1, \infty}$ and $h_{n}=h_{m}$ on $X_{n} \cap X_{m}$, for all $m, n \in \mathbb{N} \cup\{0\}$. Then,

i) if $x_{0}$ is a k-periodic point for $\left\{f_{n}\right\}$, then $h\left(x_{0}\right)$ is a k-periodic point $\left\{g_{n}\right\}$,

ii) if $x_{0}$ is a finite orbit point for $\left\{f_{n}\right\}$, then $h\left(x_{0}\right)$ is a finite orbit point for $\left\{g_{n}\right\}$.

Proof. (i) Because, $x_{0}$ is a $k$-periodic point for $\left\{f_{n}\right\}$, then $f_{m k+n}\left(x_{0}\right)=f_{n}\left(x_{0}\right)$, for every $m, n \in \mathbb{N} \cup\{0\}$. Thus,

$$
g_{m k+n}\left(h_{0}\left(x_{0}\right)\right)=h_{m k+n}\left(f_{m k+n}\left(x_{0}\right)\right)=h_{n}\left(f_{n}\left(x_{0}\right)\right)=g_{n}\left(h_{0}\left(x_{0}\right)\right) \text {, }
$$

This means that $h_{0}\left(x_{0}\right)$ is a $k$-periodic point for $\left\{g_{n}\right\}$.

(ii) Because, $x_{0}$ is a finite orbit point, then the set $\operatorname{orb}\left(x_{0}\right)$ is finite. Suppose that for indexes $i$ and $j$, we have $x_{i}=x_{j}$. Therefore, $h_{i}\left(x_{i}\right)=h_{j}\left(x_{j}\right)$. This asserts that the set $\left\{h_{n}\left(x_{n}\right) \mid n \in \mathbb{N} \cup\{0\}\right\}$ is finite. This completes the proof. 
By the hypothesis of Theorem 6, if $P\left(f_{n}\right)$ (resp. finite orbit points in GNDS $\left.f_{1, \infty}\right)$ is dense in $X_{0}$, then $P\left(g_{n}\right)$ (resp. finite orbit points in GNDS $g_{1, \infty}$ ) is dense in $Y_{0}$.

At the end of this section, let $(X, d)$ and $\left(Y, d^{\prime}\right)$ be two metric spaces and the subspace $X_{0} \subset X$ and $Y_{0} \subset Y$ without isolated points. At first we generalize the definition of sensitivity in [16].

Definition 5. Suppose that $f_{1, \infty}$ is a GNDS on $X_{0}$. Also, suppose that there exists a constant $\delta$ such that for each $x_{0} \in X_{0}$ and each open set $U_{0}$ of $x_{0}$ in $X_{0}$, there exists $y_{0} \in U_{0}$ and a positive integer $n$ such that $d\left(x_{n}, y_{n}\right)>\delta$. Then $f_{1, \infty}$ is said to have sensitive dependence on initial conditions in $X_{0}$.

In [18] it is shown that under certain conditions, transitivity and denseness of periodic points imply sensitivity. The sequence of maps $\left\{h_{n}\right\}_{n=1}^{\infty}$ is said to be uniformly equicontinuous in the set sequence $\left\{X_{n}\right\}_{n=1}^{\infty}$, if for any $\epsilon>0$ there exists a $\delta>0$ such that for all $n \in \mathbb{N}$ and for all $x, y \in X_{n}$ with $d(x, y)<\delta$, we have $d^{\prime}\left(h_{n}(x), h_{n}(y)\right)<\epsilon[15]$.

Proposition 3. Assume that GNDS $f_{1, \infty}$ is $\left\{h_{n}\right\}$-conjugate to GNDS $g_{1, \infty}$ and $\left\{h_{n}\right\}_{n=1}^{\infty}$ is uniformly equicontinuous in set $\left\{X_{n}\right\}_{n=1}^{\infty}$. If $g_{1, \infty}$ has sensitive dependence on initial conditions in $Y_{0}$, then the same is true for $f_{1, \infty}$ in $X_{0}$,

Proof. Suppose that $g_{1, \infty}$ has sensitive dependence on initial conditions in $Y_{0}$ with a sensitivity constant $\tilde{\delta}$. Since, $\left\{h_{n}\right\}_{n=1}^{\infty}$ is uniformly equicontinuous in $\left\{X_{n}\right\}_{n=1}^{\infty}$, there exists a constant $\delta>0$ such that for all $n \in \mathbb{N}$ and for all $x, y \in X_{n}$ with $d(x, y)<\delta$, we have $d^{\prime}\left(h_{n}(x), h_{n}(y)\right)<\tilde{\delta}$. Let $x_{0}$ be any point of $X_{0}$ and $U_{0}$ be any open neighbourhood of $x_{0}$. Take $y_{0}:=h_{0}\left(x_{0}\right)$ and $\tilde{U}_{0}:=h_{0}\left(U_{0}\right)$. Then $\tilde{U}_{0}$ is an open neighbourhood of $y_{0}$ since $h_{0}$ is homeomorphism. By assumption, there exist $\tilde{y}_{0} \in \tilde{U}_{0}$ and a natural number $n$ such that $d^{\prime}\left(g_{n}\left(\tilde{y}_{0}\right), g_{n}\left(y_{0}\right)\right) \geq \tilde{\delta}$. Consequently, by letting $\tilde{x}_{0}:=h_{0}^{-1}\left(\tilde{y}_{0}\right)$, we have $d^{\prime}\left(h_{n}\left(\tilde{x}_{n}\right), h_{n}\left(x_{n}\right)\right) \geq \tilde{\delta}$. This implies that $d\left(\tilde{x}_{n}, x_{n}\right) \geq \delta$ and implies the proof of the assertion.

Definition 5 leads us to extend Devaney's and Wiggins's chaos as follows:

Definition 6. GNDS $f_{1, \infty}$ is said to be chaotic in the sense of Devaney on $X_{0}$ if

1. the set of periodic points of $f_{1, \infty}$ is dense in $X_{0}$,

2. $f_{1, \infty}$ is topologically transitive on $X_{0}$,

3. $f_{1, \infty}$ has sensitive dependence on initial condition in $X_{0}$.

Also, $f_{1, \infty}$ said to be chaotic in the sense of Wiggins on $X_{0}$ if it satisfies the conditions (2) and (3).

Now assume that homeomorphisms $\left\{h_{n}\right\}_{n=1}^{\infty}$ and $\left\{h_{n}^{-1}\right\}_{n=1}^{\infty}$ are uniformly equicontinuous in the set $\left\{X_{n}\right\}_{n=1}^{\infty}$ and $\left\{Y_{n}\right\}_{n=1}^{\infty}$, respectively. Moreover, assume that $h_{m}=h_{n}$ on $X_{n} \cap X_{m}$ and $h_{m}^{-1}=h_{n}^{-1}$ on $Y_{n} \cap Y_{m}$ for all $m, n \in \mathbb{N} \cup\{0\}$. If GNDSs $g_{1, \infty}$ and $\left\{f_{n}\right\}$ are $\left\{h_{n}\right\}$-conjugate, then $f_{1, \infty}$ is chaotic in the sense of Devaney (resp. Wiggins ) on $X_{0}$, if and only if $g_{1, \infty}$ is on $Y_{0}$.

We say that the sequence of maps $\left\{h_{n}\right\}_{n=1}^{\infty}$ is uniformly $h_{0}$-equicontinuous in the set sequence $\left\{X_{n}\right\}_{n=1}^{\infty}$, if for any $\epsilon>0$ there exists a $\delta>0$ such that for all $n \in \mathbb{N}$ and for all $x \in X_{n}$ and $y \in X_{0}$ with $d(x, y)<\delta$, we have $d^{\prime}\left(h_{n}(x), h_{0}(y)\right)<\epsilon$.

Proposition 4. Assume that GNDS $\left\{f_{n}\right\}$ is $\left\{h_{n}\right\}$-conjugate to GNDS $\left\{g_{n}\right\}$ and $\left\{h_{n}\right\}_{n=1}^{\infty}$ is uniformly $h_{0^{-}}$ equicontinuous in the set $\left\{X_{n}\right\}_{n=1}^{\infty}$. Then if $x_{0}$ is a weakly hypercyclic point for $\left\{f_{n}\right\}$, then $h\left(x_{0}\right)$ is a weakly hypercyclic point for $\left\{g_{n}\right\}$.

Proof. Assume that $y_{0} \in Y_{0}$ and $\epsilon>0$ are selected arbitrarily. Since $\left\{h_{n}\right\}_{n=1}^{\infty}$ is uniformly $h_{0}$-equicontinuous in the set sequence $\left\{X_{n}\right\}_{n=1}^{\infty}$, there exists a $\delta>0$ such that for all $n \in \mathbb{N}$ and for all $x \in X_{n}$ and $y \in X_{0}$ with $d(x, y)<\delta$, we have $d^{\prime}\left(h_{n}(x), h_{0}(y)\right)<\epsilon$. By assumption, there exists $n \in \mathbb{N}$ such that $x_{n} \in \mathbb{B}_{d}\left(h_{0}^{-1}\left(y_{0}\right), \delta\right)$. Consequently, $d^{\prime}\left(h_{n}\left(x_{n}\right), y_{0}\right)<\epsilon$. The $\left\{h_{n}\right\}$-conjugacy property gives that $g_{n}\left(h_{0}\left(x_{0}\right)\right) \in \mathbb{B}_{d^{\prime}}\left(y_{0}, \epsilon\right)$ and this implies that $h_{0}\left(x_{0}\right) \in O_{2}$ in GNDS $\left\{g_{n}\right\}$. 
Consequently, if GNDS $g_{1, \infty}$ is $\left\{h_{n}\right\}$-conjugate to GNDS $\left\{f_{n}\right\}$, in addition $\left\{h_{n}\right\}_{n=1}^{\infty}$ and $\left\{h_{n}^{-1}\right\}_{n=1}^{\infty}$ are uniformly $h_{0}$-equicontinuous in the set $\left\{X_{n}\right\}_{n=1}^{\infty}$ and $\left\{Y_{n}\right\}_{n=1}^{\infty}$, respectively. Then, ${\overline{O_{2}}}^{X_{0}}=X_{0}$, if and only if ${\overline{O_{2}}}^{Y_{0}}=Y_{0}$, since $h_{0}$ is a homeomorphism.

It is natural to look for type of $\left\{h_{n}\right\}$-conjugacy to keep most of the usual dynamical properties unchanged. Above discussion leads us to pose the following definition.

Definition 7. Let $X$ and $Y$ be two metric spaces and $X_{0} \subset X, Y_{0} \subset Y$. Let $f_{1, \infty}$ and $g_{1, \infty}$ be two GNDSs on $X_{0}$ and $Y_{0}$, respectively. If there exist homeomorphisms $h_{0}: X_{0} \rightarrow Y_{0}$ and $h_{n}: X_{n} \rightarrow Y_{n}$ such that

i) $h_{n} \circ f_{n}=g_{n} \circ h_{0}$, for each $n \in \mathbb{N}$,

ii) $\left\{h_{n}\right\}_{n=1}^{\infty}$ and $\left\{h_{n}^{-1}\right\}_{n=1}^{\infty}$ are uniformly equicontinuous and uniformly $h_{0}$-equicontinuous in set $\left\{X_{n}\right\}_{n=1}^{\infty}$ and $\left\{Y_{n}\right\}_{n=1}^{\infty}$, respectively,

iii) $h_{m}=h_{n}$ on $X_{n} \cap X_{m}$ and $h_{m}^{-1}=h_{n}^{-1}$ on $Y_{n} \cap Y_{m}$ for all $m, n \in \mathbb{N} \cup\{0\}$.

Then GNDS $f_{1, \infty}$ is said to be strongly topologically $\left\{h_{n}\right\}$-conjugate to GNDS $g_{1, \infty}$.

Strongly $\left\{h_{n}\right\}$-conjugacy establishes an equivalent relation on GNDSs that shares invariant sets, weakly $k$ periodic points, periodic points, finite orbit points, transitivity, sensitive dependence on initial conditions in $X_{0}$ and the sets $O_{i}, i=1,2$.

\section{Generalized linear nonautonomous discrete systems}

In this section we discuss the case that underlying space $X$ is a Frechet space, i.e. a vector space endowed with a separating increasing sequence $\left(p_{n}\right)_{n \in \mathbb{N}}$ of seminorms which is complete with the following metric:

$$
d(x, y):=\sum_{n=1}^{\infty} \frac{1}{2^{n}} \min \left(1, p_{n}(x-y)\right), \quad x, y \in X .
$$

An important feature of this metric is that it is translation-invariant, i.e. $d(x, y)=d(x+z, y+z)$ for all $x, y, z \in X$ [5].

Definition 8. Let $X$ be a Frechet space and $X_{0}$ be a Frechet subspace of $X$. A sequence $\left\{T_{n}\right\}_{n=1}^{\infty}$ of linear maps $T_{n}: X_{0} \rightarrow X$ is called a generalized linear nonautonomous discrete system (in short GLNDS) on $X_{0}$.

At first we show that in case of the GLNDSs, sensitive dependence on initial conditions follows from the topological transitivity. Hence, one may omit sensitivity assumption (third condition) from Devaney's and Wiggins's definitions of chaos in the category of the GLNDSs.

Theorem 7. Suppose that the GLNDS $T_{1, \infty}$ is topologically transitive on $X_{0}$. Then it has sensitive dependence on initial conditions.

Proof. Let $\delta=1$ and choose $x \in X_{0}, \epsilon>0$. Also, let nonempty open sets $U_{0}:=\mathbb{B}_{d}(0, \epsilon)$ and $V_{0}:=\left\{z \in X_{0}\right.$ : $d(z, 0)>1\}$. Since $T_{1, \infty}$ is topologically transitive, there exists some $n \in \mathbb{N}$ such that

$$
T_{n}\left(U_{0}\right) \cap V_{0} \neq \phi .
$$

Thus, there exists $z \in U_{0}$ such that $T_{n}(z) \in V_{0}$. Consider $y:=x+z$. By using the translation-invariant property of the metric, we obtain

$$
d(x, y)=d(y-x, 0)=d(z, 0)<\epsilon .
$$

This means that $y \in \mathbb{B}_{d}(x, \epsilon)$. On the other hand,

$$
d\left(T_{n}(x), T_{n}(y)\right)=d\left(T_{n}(y-x), 0\right)=d\left(T_{n}(z), 0\right)>1,
$$

which implies that GLNDS has sensitive dependence on initial conditions with sensitivity constant $\delta=1$. 
The next theorem states the relation between hypercyclicity and sensitivity.

Theorem 8. Suppose $T_{1, \infty}$ is hypercyclic GLNDS. Then it has sensitive dependence on initial conditions.

Proof. Let $\delta=1$, and choose $z_{0}$ be a point that $\operatorname{orb}\left(z_{0}\right) \cap X_{0}$ is dense in $X_{0}$. Let $x_{0} \in X_{0}$ and $U_{0}$ be an open set in $X_{0}$ containing $x_{0}$. Then there exists a non zero number $\alpha$ small enough such that $\alpha z_{0}+x_{0}$ belongs to $U_{0}$. Now let $y_{0}:=\alpha z_{0}+x_{0}$. Hence $d\left(x_{n}, y_{n}\right)=d\left(\alpha z_{n}, 0\right)$ for all $n \in \mathbb{N}$. We can select the natural number $n$ large enough, such that $d\left(\alpha z_{n}, 0\right)>\delta$. Thus, $d\left(x_{n}, y_{n}\right)>\delta$, that implies it has sensitive dependence on initial conditions.

By a small change in the proof of Theorem 8 , it can be proved that the existence of a weakly hypercyclic point always implies the sensitivity.

Now we bring two examples of GLNDSs stated in the iterative way to show that the inverse of Theorems 1 and 2 is not always true. Next example is topologically transitive while $\overline{O_{1}} \neq X_{0}$. Furthermore, $O_{1}=\emptyset$ that means it is not hypercyclic.

Example 5. Let $X=\mathbb{R}^{3}$ and $X_{0}=\mathbb{R}^{2} \times\{0\} \subseteq \mathbb{R}^{3}$. Let $0<\alpha<\frac{\pi}{4}$ be an irrational number, and $\left\{\alpha_{n}\right\}_{n=0}^{\infty}$ be a dense sequence in $\mathbb{R}^{*}$ with $\alpha_{0}=1$. Let $\left\{r_{n}\right\}_{n=0}^{\infty}$ be the dense sequence of rational numbers $r_{l}=\frac{m}{n},(m, n)=1$ in the interval $[0,1)$ which are arranged in ascending order of denominators, i.e. $\left\{r_{n}\right\}_{n=0}^{\infty}=\left\{0, \frac{1}{2}, \frac{1}{3}, \frac{2}{3}, \frac{1}{4}, \frac{3}{4}, \frac{1}{5}, \ldots\right\}$. For given $n \in \mathbb{N}$, there is exactly one $k \in \mathbb{N} \cup\{0\}$ such that $2^{k} \leq n \leq 2^{k+1}-1$. Hence, take $X_{n}$ be the plane that includes the half plane

$$
\widetilde{X}_{n}=\left\{(\rho, \theta, \psi)_{s} \mid \theta=r_{k} \pi, 0<\psi<\pi, \rho>0\right\},
$$

in which $(,,)_{s}$ denotes spherical coordinates for $\mathbb{R}^{3}$. Now, $\left\{T_{n}\right\}_{n=0}^{\infty}$ is defined as follows: consider the operator $T_{0}: X_{0} \rightarrow X_{1}$ defined by $(x, y, 0) \mapsto(x, 0, y)$ and let $T_{n}: X_{n} \rightarrow X_{n+1}$ to be defined by

$$
T_{n}(x, y, z)=\left\{\begin{array}{lc}
\frac{\alpha_{n-2^{k}+1}}{\alpha_{n-2^{k}}}(x, y, z) & 2^{k} \leq n<2^{k+1}-2 \\
(x, y, z) & n=2 \\
\frac{1}{\alpha_{2^{k}-2}}(x, y, z) & n=2^{k+1}-2 \\
R_{2}^{k} \circ R_{1}^{k}(x, y, z) & n=2^{k+1}-1
\end{array}\right.
$$

where $R_{1}^{k}: X_{2^{k+1}-1} \rightarrow X_{2^{k+1}}$ is the linear transformation that transfers the point $\left(\rho, r_{k} \pi, \psi\right)_{s} \in \widetilde{X}_{2^{k+1}-1}$ to $\left(\rho, r_{k+1} \pi, \psi\right)_{s} \in \widetilde{X}_{2^{k+1}}$. And also, $R_{2}^{k}: X_{2^{k+1}} \rightarrow X_{2^{k+1}}$ is the linear transformation that rotates the points counterclockwise by the angle $\alpha$. In other words, $R_{2}^{k}: \widetilde{X}_{2^{k+1}} \rightarrow X_{2^{k+1}}$ is given by $(\rho, \theta, \psi)_{s} \mapsto(\rho, \theta, \psi-\alpha)_{s}$. Firstly, we show that $\left\{T_{n}\right\}_{n=0}^{\infty}$ is topologically transitive. It is easy to check that $\left\{T_{n}\right\}_{n=0}^{\infty}$ satisfies the following equalities:

(i) $T_{2^{k+1}-2} \circ T_{2^{k+1}-3} \circ \cdots \circ T_{2^{k}}=i d_{X_{2^{k}}} \quad \forall k \in \mathbb{N}$,

(ii) $T_{2^{k}+m-1} \circ T_{2^{k}+m-2} \circ \cdots \circ T_{2^{k}}=\alpha_{m} i d_{X_{2^{k}}} \quad \forall 1 \leq m<2^{k}-1$.

From relation (i), we obtain:

$$
T_{2^{k-1}} \circ T_{2^{k-2}} \circ \cdots \circ T_{2} \circ T_{1} \circ T_{0}=T_{2^{k-1}} \circ T_{2^{k-1}-1} \circ \cdots \circ T_{3} \circ T_{1} \circ T_{0} .
$$

Let $U_{0}$ and $V_{0}$ be two open sets in $X_{0}$ which are bounded regions between two sectors;

$$
\left\{(x, y, 0) \mid m_{1} x<y<m_{2} x, r_{1}<x^{2}+y^{2}<r_{2}\right\} .
$$

Let $R_{\psi}$ be a $\psi$-valued counter-clockwise rotation on the points $X_{0}$. Thus, there exist $p \in U_{0}, m_{0} \in \mathbb{N}$ and $I_{p} \subset(0,1)$ such that $R_{2 \pi \theta}\left(\alpha_{m_{0}} . p\right) \in V_{0}$ for all $\theta \in I_{p}$.

We identify $[0,1] \simeq S^{1} \times\{0\}$ by $\theta \sim(\cos 2 \pi \theta, \sin 2 \pi \theta, 0)$. Then there exists $k_{0} \in \mathbb{N}$ such that for each $l \in \mathbb{N}$ we have

$$
S^{1} \subset \bigcup_{i=l}^{l+k_{0}} R_{i \alpha}\left(I_{p}\right) .
$$


Choose $l_{0}$ such that $m_{0}<2^{l_{0}}$ and

$$
\left\{r_{2^{l_{0}}}, r_{2^{l_{0}+1}}, \cdots, r_{2^{l_{0}+k_{0}}}\right\} \subset I_{p} .
$$

From relation (4.2), there exists $0 \leq j_{0} \leq k_{0}$ such that

$$
T_{2^{l_{0}+j_{0}-1}} \circ T_{2^{l_{0}+j_{0}-1}-1} \circ \cdots \circ T_{3} \circ T_{1} \circ T_{0}\left(I_{p}\right) \cap \frac{V_{0}}{\left|V_{0}\right|} \neq \phi .
$$

Here, $\frac{V_{0}}{\left|V_{0}\right|}:=\left\{\frac{x}{|x|} \mid x \in V_{0}\right\}$. By using relations (4.1), we obtain

$$
\begin{aligned}
& T_{2^{l_{0}+j_{0}+m_{0}-1}} \circ T_{2^{l_{0}+j_{0}+m_{0}-2}} \circ \cdots \circ T_{2} \circ T_{1} \circ T_{0}\left(U_{0}\right) \cap V_{0} \\
= & T_{2^{l_{0}+j_{0}+m_{0}-1}} \circ \cdots \circ T_{2^{l_{0}+j_{0}}} \circ T_{2^{l_{0}+j_{0}-1}} \circ T_{2^{l_{0}+j_{0}-1}-1} \circ \cdots \circ T_{3} \circ T_{1} \circ T_{0}\left(U_{0}\right) \cap V_{0} \\
= & T_{2^{l_{0}+j_{0}-1}} \circ T_{2^{l_{0}+j_{0}-1}-1} \circ \cdots \circ T_{3} \circ T_{1} \circ T_{0}\left(\alpha_{m_{0}} U_{0}\right) \cap V_{0} \\
\neq & \emptyset,
\end{aligned}
$$

since $R_{2 \pi \theta}\left(\alpha_{m_{0}} \cdot U_{0}\right) \cap V_{0} \neq \emptyset$, for all $\theta \in I_{p}$ and $T_{2^{l_{0}+j_{0}-1}} \circ T_{2^{l_{0}+j_{0}-1}-1} \circ \cdots \circ T_{3} \circ T_{1} \circ T_{0}\left(\alpha_{m_{0}} U_{0}\right)$ intersects $R_{2 \pi \theta}\left(\alpha_{m_{0}} . U_{0}\right)$, vertically. We emphasis that our selection of $l_{0}$ ensures that the plane $X_{2^{l_{0}+j_{0}}}$ intersects the set $V_{0}$ and $2^{l_{0}+j_{0}} \leq 2^{l_{0}+j_{0}}+m_{0}<2^{l_{0}+j_{0}+1}$. Therefore, $\left\{T_{n}\right\}_{n=0}^{\infty}$ is topologically transitive. On the other hand, we obtain $O_{1}=\emptyset$. To see this, assume that $q \in \mathbb{R}^{2} \times\{0\}$ and for any $n \in \mathbb{N}$

$$
T_{n} \circ T_{n-1} \circ \cdots T_{0}(q) \in\left(\mathbb{R}^{2} \times\{0\}\right) \cap X_{n+1} .
$$

Thus when the orbit of $q$ leaves the plane $X_{n+1}$, it can not again intersects $\mathbb{R}^{2} \times\{0\}$ since $\alpha$ is an irrational number. This tells us that $\operatorname{orb}(q) \cap\left(\mathbb{R}^{2} \times\{0\}\right)$ is a finite set with cardinality smaller than $2^{k}+1$.

Note that, the GNDS appeared in Example 5 is chaotic in the sense of Wiggins but not in the sense of Devaney. The GLNDS appeared in the next example satisfies $\overline{\mathrm{O}_{2}}=X_{0}$, while it is not topologically transitive, see [12].

Example 6. Let $X=\mathbb{R}^{2}, X_{0}=\mathbb{R} \times\{0\}$ and $X_{n}=\left\{\left(x, \frac{x}{n}\right): x \in \mathbb{R}\right\}$ for each $n \in \mathbb{N}$. Consider $T_{0}: X_{0} \rightarrow X_{1}$ given by $(x, 0) \mapsto(x, x)$, and $T_{n}: X_{n} \rightarrow X_{n+1}$ given by $(x, y) \mapsto \frac{r_{n+1}}{r_{n}}\left(x, \frac{n y}{n+1}\right)$ where $\left\{r_{n}\right\}_{n=1}^{\infty}$ is a dense sequence of rational non zero numbers. It is not hard to show that $\left\{T_{n}\right\}_{n=0}^{\infty}$ is not topologically transitive, since $X_{n} \cap X_{0}=\{(0,0)\}$ for all $n \in \mathbb{N}$. Moreover, $\overline{O_{2}}=X_{0}$ since

$$
T_{n} \circ T_{n-1} \circ \cdots T_{0}(x, 0)=\frac{r_{n+1}}{r_{1}}\left(x, \frac{1}{n+1} x\right),
$$

for all $n \in \mathbb{N}$. Note that, $O_{2}=X_{0}-\{(0,0)\}$ and so is a $G_{\delta^{-}}$set.

Acknowledgment: The author thanks Neda Abbasi who assisted in writing the paper.

\section{References}

[1] Z. AlSharawi, J. Angelos, S. Elaydi, L. Rakesh, An extension of Sharkovskys theorem to periodic difference equations. J. Math. Anal. Appl, 2006, 316, 128-141.

[2] F. A. B. Coutinho, M. N. Burattini, L. F. Lopez, E. Massad, Threshold conditions for a non-autonomous epidemic system describing the population dynamics of dengue. Bull Math Biol, 2006, 68, 2263-2282.

[3] J. Dvorakova, Chaos in nonautonomous discrete dynamical systems. Commun Nonliear Sci Numer Simulat, 2012, 17, 46494652.

[4] G. L. Forti, Various notion of chaos for discrete dynamical systems. Aequationes Math, 2005, 70, 1-13.

[5] K. G. Grosse-Erdmann, A. Peris Manguillot, Linear chaos. Springer, 2011.

[6] P. Kloeden, M. Rasmussen, Nonautonomous dynamical Systems. Amer Mathematical Society, 2011. 
[7] S. Kolyada, M. Misiurewicz, L. Snoha, Topological entropy of nonautonomous piecewise monotone dynamical systems on the interval. Fund. Math. 160 (1999).

[8] L. Moreau, Stability of multiagent systems with time-dependent communication links. IEEE Transactions On Automatic Control, 2005, 50, 169-182.

[9] P. Oprocha, A quantum harmonic oscillator and strong chaos. J Phys A, Math Gen, 2006, 39, 14559-14565.

[10] C. Ptzsche, Geometric theory of discrete nonautonomous dynamical systems. Springer, 2010.

[11] M. Pourbarat, N. Abbasi, Nonautonomous discrete systems: density of orbits and transitivity. Int. J. Bifurcation Chaos, 2015, 25, 1550065-1550070.

[12] M. Pourbarat, N. Abbasi, On subspaces that are not Baire. New Zealand J. Math, 2015, 45, 57-61.

[13] C. Robinson, Dynamical systems: stability, symbolic dynamics and chaos. 2nd Ed. CRC Press, Florida, 1999.

[14] Y. Shi, Chaos in nonautonomous discrete dynamical systems approached by their induced systems. Int. J. Bifurcation Chaos, 2012, 22, 1250284-1250296.

[15] Y. Shi, G. Chen, Chaos of time-varying discrete dynamical systems. Journal of Difference Equations and Applications, 2009, 15, 429-449.

[16] C. Tian, G. Chen, Chaos of a sequence of maps in a metric space. Chaos Solitons Fractals, 2006, 28, 1067-1075.

[17] W. Zhang, Discrete dynamical systems. Bifurcations and Chaos in Economics, Elsevier, 2006.

[18] H. Zhu, Y. Shi, H. Shao, Devaney chaos in nonautonomous discrete systems. Int. J. Bifurcation Chaos, 2016, 26, 16501901650199. 